\title{
L'Ancien Testament et les institutions
}

Thomas Romer

Citer ce document / Cite this document :

Romer Thomas. L'Ancien Testament et les institutions. In: Autres Temps. Cahiers d'éthique sociale et politique. N61, 1999. pp. 43-48;

doi : https://doi.org/10.3406/chris.1999.2101

https://www.persee.fr/doc/chris_0753-2776_1999_num_61_1_2101

Fichier pdf généré le 26/03/2019 


\title{
L'Ancien Testament et les institutions
}

\author{
Thomas Römer*
}

La Bible hébraïque présente les institutions de manière ambiguë. On y trouve à la fois la mise en place du culte sacrificiel à pratiquer au temple et l'annonce de la destruction du temple. À la royauté davidique est promise une dynastie éternelle ( $2 S 7$ ), mais l'exil babylonien va mettre un terme à la monarchie judéenne. Quant au clergé, sa médiation paraît indispensable selon certains textes, selon d'autres, contestable, voire superflue.

Il n'est donc guère étonnant que l'Ancien Testament ait été utilisé à la fois pour légitimer les pouvoirs ecclésiastique et politique en place, et pour contester ces mêmes pouvoirs dans le cadre d'une lecture prophétique. Au lieu de nous cantonner dans cette alternative, nous voudrions nous interroger sur l'ambiguiité exprimée par les textes bibliques vis-à-vis des institutions.

\section{Les institutions en Israël et en Juda à l'époque de la monarchie}

Les royaumes d'Israël et de Juda fonctionnent sur le même modèle institutionnel qui régit les sociétés du Proche-Orient ancien : un roi, une administration de cour, un clergé, un temple et des sanctuaires locaux. L'identité du peuple s'exprime à travers la vénération d'un Dieu national : Yahvé dans le Nord, et dans le Sud peut-être El Elyon jusqu'à la « récupération » de Yahvé à l'époque du roi Josias. Il n'y a point de séparation entre le religieux, l'économique et le politique. Le roi est « fils de Dieu » et contrôle, au moins théoriquement, le clergé, les impôts et la jurisprudence. Certes, il y a toujours des cercles contestataires de l'institution monarchique, notamment auprès de l'aristocratie rurale, qui exprime naturellement une certaine hostilité à l'égard de la centralisation du pou-

* Thomas Römer est professeur d'Ancien Testament à la faculté de théologie de l'Université de Lausanne (Suisse). 
voir étatique. La « loi royale » exposée par Samuel (1 S 8), illustre bien les réticences face aux institutions de la royauté, même si ce texte n'a été rédigé qu'après l'effondrement de celle-ci. Les institutions de la royauté coûtent cher, aussi bien au niveau fiscal qu'au niveau de la restriction des libertés individuelles. Quelques prophètes, comme Amos et Osée, critiquent les abus qui détériorent la cohésion sociale, mais la royauté ou le temple ne sont pas remis en question en tant que tels, même dans les oracles aussi durs que celui de Am 5,21-24 : ce que Dieu déteste, c'est un culte qui se désintéresse du droit et de la justice. Cette insistance sur le droit et la justice, sur la défense de la veuve et de l'orphelin, n'est ni une invention ni une spécificité du discours prophétique. En effet, selon l'idéologie royale proche-orientale, la tâche suprême du roi est le maintien de la cohésion sociale, ce qui implique de faire attention à ceux qui sont en marge de la société (Ps 72,12-14). L'ordre du monde tel qu'il a été voulu par le Dieu créateur doit donc se refléter dans l'organisation de la société. Les différentes institutions sont au service de la réalisation de ce projet.

La mise en question radicale des institutions liées à la monarchie n'est pas par conséquent un phénomène interne aux sociétés israélite et judéenne ; elle est provoquée par des événements externes.

\section{Centralisation et mise en question des institutions de la royauté}

La destruction de Samarie par les Assyriens et la transformation du royaume d'Israël en province assyrienne ont dû être ressenties comme un choc par le voisin judéen. Mais en même temps le malheur du "grand frère » du Nord faisait le bonheur du petit royaume du Sud. L'archéologie atteste de l'énorme essor économique dont bénéficie Jérusalem vers la fin du VIII' siècle av. J.-C. Le royaume de Juda était libéré de sa dépendance vis-à-vis du royaume d'Israël et même les Assyriens avaient commencé à lâcher prise. En 701 av. J.-C. ils abandonnent, pour des raisons qui restent mystérieuses, le siège de Jérusalem, et quelques décennies plus tard ils doivent réduire fortement leur présence en Syrie-Palestine afin de défendre l'empire contre les Babyloniens. C'est dans ce contexte qu'à lieu, à Jérusalem, une « réforme » des institutions entreprise par le roi Josias.

Selon le récit biblique, Josias n'a que huit ans lorsqu'il devient roi. Il est soutenu par une partie du " peuple du pays », c'est-à-dire l'aristocratie rurale, qui pour l'occasion s'est liée au parti réformiste de la cour de Jérusalem. La réforme dont parle le récit de 2 R 22-23 consiste en effet 


\section{L'Ancien Testament et les institutions}

en une plus grande centralisation des institutions à Jérusalem. Les sanctuaires locaux sont supprimés, leur clergé se trouve au chômage. La centralisation du culte étatique bouleverse quelques institutions traditionnelles. Du fait qu'il n'y a plus qu'un seul sanctuaire licite, l'abattage profane, impensable dans une société rurale, devient possible, et du coup le clergé n'est plus une institution indispensable (cf. Dt 12).

Par la centralisation du culte et de la fiscalité à Jérusalem, par l'uniformisation du culte de Yahvé, la réforme de Josias semble avoir en vue le renforcement de l'autorité royale. Or, paradoxalement, le livre du Deutéronome, qui dans sa première version était sans doute destiné à accompagner et légitimer ces changements institutionnels, ne parle quasiment pas du souverain, à l'exception de la loi sur le roi (Dt 17,14-20), texte dont l'appartenance au Deutéronome primitif est fortement discutée. Et ce texte dit surtout ce que le roi ne doit pas avoir : pas trop de richesses, pas trop de femmes. Ce qu'il doit surtout et exclusivement faire, c'est s'occuper de la loi de Yahvé.

Comment interpréter cette restriction du pouvoir royal ? S'agit-il d'une indication que le vrai pouvoir, sous Josias, est dans la main de ses conseillers (les «Deutéronomistes »), auteurs de la première édition du Deutéronome ; ou avons-nous affaire à un texte qui n'a été écrit qu'après la disparition de la royauté judéenne?

De toute façon, l'époque de Josias était seulement un dernier sursaut avant l'écroulement des institutions de la monarchie.

La destruction de Jérusalem par les Babyloniens et la déportation de l'intelligentsia (597 et 586) signifient le démantèlement total des institutions traditionnelles. La destruction du temple, l'abolition de la monarchie davidique, la dispersion géographique des Judéens (Palestine, Babylone, Égypte) avaient détruit la cohésion sociale. Cette crise de l'époque babylonienne amena les intellectuels judéens de redéfinir les institutions de jadis.

\section{L'espace du temple devient l'espace du livre}

Les prophètes préexiliques avaient critiqué certains aspects du culte célébré dans les sanctuaires locaux, mais ils n'avaient pas envisagé la disparition du temple en tant que lieu de rencontre entre Dieu et les hommes. La destruction du sanctuaire central avait mis en question la fonction du temple comme espace sacré. Après les événements de 587, les descendants des anciens fonctionnaires royaux (les «Deutéronomistes ») opérèrent 
alors un déplacement qui devint fondamental pour le judaïsme. En racontant l'histoire de la royauté, ils consacrent une place importante à la construction du temple par Salomon, dont l'apothéose se trouve dans la prière d'inauguration en $1 \mathrm{R} 8$. Or, ce long discours royal amène les destinataires progressivement à un éloignement du temple. Le texte met en place plusieurs occasions de prière. Dans les premières occasions, le lieu de supplication se trouve dans la Maison c'est-à-dire le temple (v. 33) ; dans le dernier scénario de prière, le peuple se trouve déporté de son pays, et la dernière invocation se fait «en direction du pays, en direction de la ville et en direction de la Maison » (v. 48). Le temple reste le lieu de l'orientation de la prière (une sorte de qibla), mais on peut prier le Dieu d'Israël dans un pays étranger. D'ailleurs $1 \mathrm{R} 8$ insiste, comme un leitmotiv, sur le fait que Dieu habite dans le ciel et non dans le temple.

La réécriture de la réforme de Josias au $\mathrm{VI}^{e}$ ou $\mathrm{V}^{\mathrm{e}}$ siècle av. notre ère franchit un pas supplémentaire dans cette direction. Dans sa forme actuelle, le récit de 2 R 22-23 ne décrit pas une réforme du temple mais un «vidage », comme l'a montré notamment Françoise Smyth-Florentin. Dans son grand ménage de purification rituelle, Josias ne fait rien d'autre que casser, brûler, démolir et supprimer (les mots-clé en $2 \mathrm{R} 23$ ). À la fin, le temple est totalement vide et lorsque le narrateur relate la Pâque qui conclut la Réforme, il n'est question ni de sacrifices, ni de prêtres, ni d'autels.

Selon 2 R 22 les grands bouleversements autour du temple sont initiés par la découverte d'un rouleau contenant les paroles de Yhwh. On l'a souvent remarqué, le récit de découverte du livre imite le motif procheoriental des tablettes de fondation. Tout roi assyrien ou babylonien se devait de rénover ou reconstruire les sanctuaires de ses prédécesseurs. La légitimation de ces changements était fournie par des « récits de découverte » dans lesquels le roi ou ses fidèles trouvent, soit par hasard soit à la suite de longues recherches, la pierre de fondation contenant le plan du sanctuaire qui correspond, bien évidemment, aux projets architecturaux du roi. $2 \mathrm{R} 22$ reprend clairement ce motif, tout en le transformant. En effet, Josias ne découvre plus le plan du temple, mais le livre de la Loi. Pour le dire autrement : l'institution du temple a été « remplacée » par le livre, nouveau médiateur entre Dieu et le peuple.

Un phénomène comparable se trouve dans l'actualisation exilique, voire postexilique, de Dt 6 . On y lit au verset $9:$ : Tu inscriras [les commandements] sur les poteaux de ta maison et sur tes portes $»$. Traditionnellement, des recommandations pour le fidèle se trouvent gravées à l'entrée ou sur les murs des sanctuaires. Dt 6,9 insinue du coup que 


\section{L'Ancien Testament et les institutions}

n'importe quelle maison peut devenir «sanctuaire », lieu de lecture de la Tora. Nous avons ici, au moins virtuellement, la naissance de l'institution du culte synagogal qui va entrer en concurrence sérieuse avec le temple. Grâce au Perses, le temple a pu être reconstruit vers 520 av. J.-C. ; mais c'est la synagogue qui permet de rassembler tout le judaïsme, celui dans le pays et celui dans la diaspora.

\section{Des institutions pour un judaïsme en dispersion}

Entre 400 et 350 av. notre ère, de nouveau avec l'appui des Perses, le Pentateuque, la Tora, devient le document qui fait autorité pour tout le judaîsme («Samaritains » inclus). Le Livre devient, comme l'a dit un exégète, la «patrie portative ». À sa manière, le Pentateuque tient compte de la disparition des institutions étatiques. Israël devient le peuple de Yahvé non pas dans le pays, mais «dans le désert » (Ex 19), le temple statique est transformé en sanctuaire mobile (Ex 25-40), et les fonctions royales sont récupérées par Moïse. Le récit de sa naissance et de son exposition (Ex 2) est quasiment un calque littéraire d'une histoire relatant la mise au monde de Sargon, fondateur de la royauté assyrienne. Dans la suite, Moïse devient l'intermédiaire privilégié à travers lequel Dieu fait connaître ses volontés. Moïse est également juge suprême (Ex 18) et Dieu l'a « établi sur toute la maison d'Israël » (Nb 12), à la manière de la vocation attribuée à la dynastie davidique en Samuel et Rois.

Au centre du Pentateuque (c'est-à-dire dans le Lévitique) se trouvent les lois rituelles et sacrificielles, ce qui montre bien l'importance du milieu sacerdotal au moment de la publication de la Tora. Après 515, le temple et son clergé redeviennent, dans la province de Judée, une institution qui fonctionne, contrairement à la royauté. Il suffit de lire la réécriture des livres Sam et Rois dans les Chroniques pour se rendre compte des transferts de pouvoir. Dans Chr les campagnes militaires se transforment en processions liturgiques. Les rois ressemblent en quelque sorte à des prêtres et le clergé apparaît comme l'institution centrale de toute l'histoire d'Israël.

Pour le milieu sacerdotal, les sacrifices ne sont possibles que dans le cadre du temple ; c'est pour cela que dans le Pentateuque les lois sur les sacrifices sont précédées par la construction du sanctuaire (Ex 25-40). Mais les juifs de la diaspora ne pouvaient guère se permettre le luxe de voyages fréquents à Jérusalem. L'institution du temple, dès l'époque perse, ne peut plus rassembler tout le judaïsme. Ainsi les auteurs sacerdo- 
taux proposent-ils, eux-mêmes, dans les deux premiers livres du Pentateuque, des institutions mobiles: Gn 1 inscrit le Sabbat dans le projet créationnel de Dieu, l'ébauche des règles alimentaires (interdiction de consommer du sang) est déjà donnée à Noé après le déluge (Gn 9), la circoncision est définie comme signe de l'alliance que Dieu a établie avec Abraham (Gn 17), et le rituel de la Pâque n'est pas institué au Sinaii, mais au moment de la sortie d'Égypte, comme fête familiale, sans la présence des prêtres (Ex 12). Sabbat, circoncision, Pâque et Kashrout deviennent dès lors des institutions qui sont gérables partout et qui ont leur autonomie par rapport aux institutions étatiques et cléricales. Et c'est cette flexibilité qui a permis au judaïsme de se dire et de se redire à travers les siècles.

\section{De l'ambiguiité des institutions}

La Bible hébraïque ne rejette nullement les institutions politiques et religieuses en vogue au premier millénaire av. notre ère. Mais suite aux événements de 597/87, elle les « désacralise », ou plutôt elle les déplace et les décloisonne. Ce ne sont plus des institutions politico-religieuses précises qui fournissent la base d'une définition du peuple de Yahvé. Le vrai enjeu, c'est dès lors de réinventer pour chaque situation nouvelle les institutions adéquates.

T. R.

\section{BIBLIOGRAPHIE}

R. de Vaux, Les institutions de l'Ancien Testament, Paris : Cerf, 1967.

F. Smyth, "Quand Josias fait son œuvre ou le roi bien enterré. Une lecture synchronique de $2 \mathrm{R} 22,1-23,28$ ", in Israël construit son histoire. L'historiographie deutéronomiste à la lumière des recherches récentes, Le Monde de la Bible 34, A. de Pury, T. Römer et J.-D. Macchi (éd.), Genève : Labor et Fides, 1996, pp. 325-339.

T. Römer, «L'Ancien Testament. Une littérature de crise », RThPh 127/4, 1995, pp. 321-338. 